\title{
RDUS
}

Revue de DROIT

UNIVERSITÉ DE SHERBROOKE

Titre : $\quad$ PRÉJUDICE MATÉRIEL, CORPOREL ET MORAL : VARIATIONS SUR LA CLASSIFICATION TRIPARTITE DU PRÉJUDICE DANS LE NOUVEAU DROIT DE LA RESPONSABILITÉ

Auteur(s): $\quad$ Nathalie VÉZINA

Revue : $\quad$ RDUS, 1993-1994, volume 24, numéro 1

Pages: $\quad 161-180$

ISSN : $\quad$ 0317-9656

Éditeur : $\quad$ Université de Sherbrooke. Faculté de droit.

URI : $\quad$ http://hdl.handle.net/11143/13365

DOl : $\quad$ https://doi.org/10.17118/11143/13365 
Page vide laissée intentionnellement. 


\section{PRÉJUDICE MATÉRIEL, CORPOREL ET MORAL : VARIATIONS SUR LA CLASSIFICATION TRIPARTITE DU PRÉJUDICE DANS LE NOUVEAU DROIT DE LA RESPONSABILITÉ}

par Nathalie VÉZINA*

Traditionnellement, lorsqu'une victime demandait réparation en vertu des règles de la responsabilité civile, le préjudice subi était partagé entre deux catégories: le préjudice était matériel ou moral, selon la nature patrimoniale ou extrapatrimoniale de l'atteinte. Dans le nouveau Code civil du Québec, les articles clés du droit de la responsabilité civile envisagent le préjudice selon trois catégories. Ce faisant, le législateur québécois opte explicitement pour la position, déjà adoptée par une partie de la jurisprudence et de la doctrine, selon laquelle le préjudice corporel doit être considéré comme étant distinct des deux catégories précédentes. L'auteure constate que cette classification entraîne des conséquences plus importantes que dans le Code civil du Bas Canada, puisque plusieurs dispositions ont une portée différente selon la nature du préjudice subi par la victime. Par ailleurs, elle considère certaines des difficultés que pourrait engendrer l'application de cette classification dans le droit nouveau.

*. Avocate et professeure à la Faculté de droit de l'Université de Sherbrooke. 
Traditionally, when a victim sought damages, the injury would be categorized as material or moral, depending on whether a patrimonial interest was involved. Under the new Civil Code of Quebec, key articles relating to civil liability divide injuries into three separate categories. In so doing, the Quebec Legislator confirms a jurisprudential and doctrinal trend which considers bodily injury as distinct from the two categories mentioned above. The writer notes that this classification will have a greater impact on the rules concerning civil liability than that which existed under the Civil Code of Lower Canada, since several articles apply differently depending on the nature of the injury suffered by the victim. In addition, she examines some of the difficulties that may arise in the application of this newly recognized classification. 
Préjudice matériel, corporel et moral :

(1993) 24 R.D.U.S. variations sur la classification tripartite du préjudice

\section{SOMMAIRE}

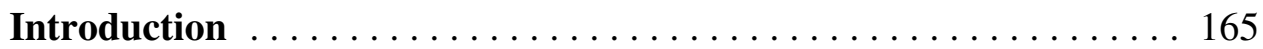

I. Évolution de la classification du préjudice . . . . . . . 165

II. Impacts de la classification du préjudice dans le nouveau droit de la responsabilité $\ldots \ldots \ldots \ldots \ldots \ldots \ldots \ldots \ldots \ldots$

III. Difficultés possibles en matière de classification du préjudice
A. Préjudice corporel et préjudice moral : double standard ou confusion des termes? ................ 175
B. La victime médiate d'une atteinte à l'intégrité corporelle subit-elle un préjudice «corporel»? . . . . . . . . . . . 177
C. Classification tripartite et législation québécoise ..... 182

Conclusion $\ldots \ldots \ldots \ldots \ldots \ldots \ldots \ldots \ldots \ldots \ldots \ldots \ldots \ldots \ldots$ 
Préjudice matériel, corporel et moral : variations sur la classification tripartite du préjudice $\quad$ (1993) 24 R.D.U.S. dans le nouveau droit de la responsabilité 
(1993) 24 Rréjudice matériel, corporel et moral :

(1993) 24 R.D.U.S. variations sur la classification tripartite du préjudice

\section{Introduction}

Il est des mots auxquels on attache, au fil du temps, une certaine signification. Dans le domaine juridique, cet usage peut toutefois être perturbé par des développements législatifs, jurisprudentiels ou même doctrinaux. Dès lors, les réflexes acquis avec les années peuvent prendre un certain temps à s'estomper, d'où l'intérêt de faire le point ${ }^{1}$.

La question de la classification du préjudice dans le droit québécois de la responsabilité justifie, selon nous, un tel exercice. Habitués à une classification «bipartite» du préjudice, les juristes québécois ont été témoins d'un mouvement graduel vers une classification «tripartite», laquelle vient d'être formellement reconnue par le législateur dans les articles cardinaux du droit de la responsabilité civile (art. 1457, 1458 et 1607 C.c.Q.).

Nous chercherons, dans un premier temps, à retracer l'évolution de la classification du préjudice et à faire le point sur la signification de chacune des catégories reconnues par le législateur québécois (I). Ceci nous amènera, dans un deuxième temps, à considérer l'importance de cette classification dans le nouveau droit de la responsabilité (II). Enfin, nous aborderons brièvement certaines difficultés qui pourraient se présenter, compte tenu des dispositions législatives présentement en vigueur (III).

\section{I. Évolution de la classification du préjudice}

Confrontées au quasi-mutisme du législateur sur la question, la jurisprudence et la doctrine ont longtemps abordé le préjudice à travers une classification bipartite : le préjudice indemnisable était soit matériel, soit moral.

En ramenant ces deux notions à leur plus simple expression, le préjudice matériel constitue une atteinte à un droit patrimonial, alors que le préjudice moral résulte d'une atteinte à un droit extrapatrimonial. La même idée est d'ailleurs suggérée en opposant préjudices «patrimonial» et «extrapatrimonial», «pécuniaire» et «non pécuniaire», «économique» et «non économique», etc ${ }^{2}$.

1. Un auteur, à l'occasion des travaux de l'Office de révision du Code civil, en a démontré l'utilité en rapport avec la résolution de contrat et certaines notions connexes - devançant en cela les efforts de clarification du législateur (art. 1604 al. 1 in fine C.c.Q.) : A. Popovici, «Notes de terminologie juridique autour de la notion de résolution de contrat» (1970-71) 73 R. du N. 343.

2. P.-A. Crépeau et autres, Dictionnaire de droit privé et lexiques bilingues, $2^{\mathrm{e}}$ éd., Cowansville, Yvon Blais, 1991, $v^{i s}$ «dommage extrapatrimonial», «dommage patrimonial», «dommage matériel» et «dommage moral»; M. Tancelin, Des obligations. L'acte illégitime et les modes d'exécution, Montréal, Wilson et Lafleur, 1993, n ${ }^{5} 55 ;$ J. Carbonnier, Droit civil, t. 4, Les obligations, $16^{\mathrm{e}}$ éd., Paris, Presses universitaires de France, 1992, nº 206; H. 
Essentiellement, la classification bipartite du préjudice correspond à la grande division des droits, c'est-à-dire les droits patrimoniaux et extrapatrimoniaux ${ }^{3}$.

Jusque là, la classification bipartite du préjudice semble dépourvue d'ambiguïtés. Les risques de glissements n'en sont pas moins présents. Ainsi, on peut être tenté d'assimiler préjudice matériel et atteinte aux biens. Le préjudice moral, par opposition, serait synonyme d'atteinte à la personne. Cette reformulation appelle pourtant des nuances importantes. En effet, il semble y avoir là une certaine confusion entre l'événement à l'origine du préjudice et le préjudice lui-même. Or, une telle assimilation s'avère erronée dans certains cas. Ainsi, une atteinte à la personne peut entraîner un préjudice matériel, c'est-à-dire qu'elle peut amener des conséquences proprement pécuniaires. Par exemple, une atteinte à la réputation ou à la vie privée peut être à l'origine de pertes financières pour la personne qui en est victime ${ }^{4}$. Inversement, une atteinte aux biens pourrait occasionner, outre un préjudice à caractère patrimonial, un préjudice extrapatrimonial (animal pour lequel la victime éprouvait une affection réelle, oeuvre dont la mutilation porte atteinte aux sentiments de

Lalou, Traité pratique de la responsabilité civile, $6^{\mathrm{e}}$ éd. par P. Azard, Paris, Dalloz, 1962, $\mathrm{n}^{\mathrm{o}} 149 ; \mathrm{Ph}$. Le Tourneau, La responsabilité civile, $3^{\mathrm{e}}$ éd., Paris, Dalloz, 1982, $\mathrm{n}^{\circ} 562 ; \mathrm{Ph}$. Malaurie et L. Aynès, Cours de droit civil, t. VI, Les obligations, 4 éd., Paris, Cujas, 1993, $\mathrm{n}^{\mathrm{os}}$ 241-247; Ph. Malinvaud, Droit des obligations, $5^{\mathrm{e}}$ éd., Paris, Litec, 1990, $\mathrm{n}^{\circ} 211 ; \mathrm{G}$. Marty et P. Raynaud, Les obligations, t. 1, Les sources, $2^{\mathrm{e}}$ éd., Paris, Sirey, 1988, $\mathrm{n}^{\text {os }} 430$ 432; H. et L. Mazeaud et A. Tunc, Traité théorique et pratique de la responsabilité civile délictuelle et contractuelle, $6^{\mathrm{e}}$ éd., t. I, Paris, Montchrestien, 1965, $\mathrm{n}^{\mathrm{o}}$ 293; H., L. et J. Mazeaud, Leçons de droit civil, t. II, vol. 1, Obligations. Théorie générale, 8 éd. par F. Chabas, Paris, Montchrestien, 1991, n 410; R. Savatier, Traité de la responsabilité civile en droit français, t. II, Paris, Librairie générale de droit et de jurisprudence, 1951, $\mathrm{n}^{\circ} 525$; B. Starck, Obligations, vol. 1, Responsabilité délictuelle, $4^{\mathrm{e}}$ éd. par H. Roland et L. Boyer, Paris, Litec, 1991, $\mathrm{n}^{\text {os }} 98$ et 106; G. Viney, Traité de droit civil, t. IV, Les obligations. La responsabilité civile: conditions, Paris, Librairie générale de droit et de jurisprudence, 1982, $\mathrm{n}^{\text {os }} 250$ et s. Voir aussi Ch. Larroumet, Droit civil, t. III, Les obligations. Le contrat, Paris, Economica, $1990, \mathrm{n}^{\text {os }} 649$ et $\mathrm{s}$.

3. Lalou, ibid., $\mathrm{n}^{\circ} 149$.

4. Le professeur Tancelin, dans la quatrième édition de son ouvrage, favorisait une classification en fonction des atteintes à la personne et aux biens; il prenait néanmoins garde d'apporter des réserves quant aux possibles incidences économiques d'une atteinte à la personne: M. Tancelin, Des obligations. Contrat et responsabilité, $4^{\mathrm{e}}$ éd., Montréal, Wilson et Lafleur, 1988, $\mathrm{n}^{\circ}$ 556. Voir aussi Y. Chartier, La réparation du préjudice dans la responsabilité civile, Paris, Dalloz, $1983, \mathrm{n}^{\text {os }} 142$ et $\mathrm{s}$.; Marty et Raynaud, supra, note $2, \mathrm{n}^{\text {os }}$ 427bis-429; Mazeaud et Tunc, supra, note 2, n 294. 
(1993) 24 Rréjudice matériel, corporel et moral :

(1993) 24 R.D.U.S. variations sur la classification tripartite du préjudice

l'artiste, objet ayant une valeur sentimentale, etc. $)^{5}$, quoique la doctrine soit plus prudente à ce sujet ${ }^{6}$.

Si l'on fait abstraction de ce risque de confusion, la classification bipartite s'avère somme toute relativement simple. Son défaut majeur réside plutôt dans son caractère rudimentaire. En effet, avec le développement des règles relatives aux atteintes à l'intégrité corporelle, la classification bipartite a révélé ses insuffisances. Il a semblé particulièrement inapproprié de placer sur un même plan les pertes pécuniaires au sens strict (par exemple, frais de remplacement ou de remise en état d'un bien, pertes d'exploitation, etc.) et celles attribuables à une atteinte à l'intégrité corporelle (perte de capacité de gains et dépenses liées aux soins). De la même façon - quoique l'assimilation puisse paraître moins choquante - il était difficile de traiter de façon identique les aspects non pécuniaires résultant d'atteintes à l'intégrité corporelle (préjudice esthétique, souffrances, perte de jouissance de la vie) et le préjudice non pécuniaire survenu indépendamment d'une telle atteinte. Une catégorie propre au préjudice corporel, c'est-à-dire portant sur les pertes pécuniaires et non pécuniaires découlant d'une atteinte à l'intégrité corporelle ${ }^{7}$, s'imposait donc d'elle-même.

5. Rares sont les autorités québécoises sur la question. Le préjudice moral pour la perte d'un animal a été évoqué de façon favorable dans Régnier c. Gosselin, [1978] C.P. 222.

La jurisprudence française a été appelée plus fréquemment à reconnaître l'existence d'un préjudice moral pour la perte d'un animal : Civ. $1^{\mathrm{re}}, 16$ janvier 1962, J.C.P. 1962.II.12557, note P. Esmein, D.1962.J.199, note R. Rodière, S.1962.J.281, note C.-I. Foulon-Piganiol, Rev. trim. dr. civ. 1962.316, obs. A. Tunc; T.G.I. Caen, 30 oct. 1962, J.C.P.1962.II.12954, D.1963.J.92, S.1963.J.152, Rev. trim. dr. civ. 1963.93, obs. A. Tunc; T.G.I. Bordeaux, 13 juil. 1984, Gaz. Pal. 1984.2 ${ }^{\mathrm{e}}$ sem.Somm.445; Rouen, 16 sept. 1992, D.S.1993.J.353, note J.-P. Marguénaud. Voir aussi Civ. 1 ${ }^{\text {re }}, 27$ janv. 1982, J.C.P.1983.II.19923, note Chabas, D.1983.I.R.500, obs. Penneau. Elle montre toutefois une moins grande ouverture dans le cas d'objets inanimés : Trib. corr. Le Mans, 14 oct. 1966, Gaz. Pal.1967.1 ${ }^{\text {er }}$ sem.J.29.

6. Certains auteurs se montrent hostiles à la reconnaissance d'un préjudice moral pour atteinte à un bien : Chabas, note sous Civ. $1^{\text {re }}, 27$ janv. 1982, ibid.; Chartier, supra, note 4, ${ }^{\circ} 159$; Esmein, note sous Civ. $1^{\text {re }}, 16$ janv. 1962, ibid.; Foulon-Piganiol, note sous Civ. $1^{\text {re }}, 16$ janv. 1962, ibid.; Le Tourneau, supra, note 2, n 576; Malaurie et Aynès, supra, note 2, no 247; R. Nerson, «La condition de l'animal au regard du droit», D.1963.Chron.1, p. 4; Tunc, obs. sous Civ. $1^{\text {re }}, 16$ janv. 1962, ibid.; idem, obs. sous T.G.I. Caen, 30 oct. 1962, ibid.; A. Weill et F. Terré, Droit civil. Les obligations, $4^{\mathrm{e}}$ éd., Paris, Dalloz, 1986, n 612 ; note sous Trib. corr. Le Mans, 14 oct. 1966, ibid.

D'autres auteurs émettent des réserves, tout en montrant une certaine ouverture : R. Rodière, note sous Civ. $1^{\text {re }}, 16$ janv. 1962, ibid.; Marguénaud, note sous Civ. $1^{\text {re }}, 16$ sept. 1992, ibid.; note sous T.G.I. Bordeaux, 13 juil. 1984, ibid.

7. On peut être tenté de parler d'atteinte à l'intégrité physique : Dictionnaire de droit privé et lexiques bilingues, supra, note $2, v^{o}$ «dommage corporel». Pourtant, une tendance récente manifeste dans certaines lois particulières - voit la protection de l'intégrité corporelle dans un sens global, incluant à la fois l'intégrité physique et psychique de la personne. À ce sujet, voir ci-dessous, partie III.C. 
Contrairement aux deux autres catégories, le préjudice corporel est caractérisé par l'objet de l'atteinte (l'intégrité corporelle) plutôt que par la nature des intérêts affectés par cette atteinte (patrimoniaux ou extrapatrimoniaux). De fait, la catégorie du préjudice corporel a pour effet d'«exproprier» en partie les deux autres catégories de préjudice ${ }^{8}$, comme l'illustrent les schémas suivants :

\section{Classification bipartite}

\begin{tabular}{|l|l|}
\hline & \\
Préjudice matériel & Préjudice moral \\
\hline
\end{tabular}

\section{Classification tripartite}

\begin{tabular}{|l|l|l|}
\hline \multirow{2}{*}{ Préjudice matériel } & $\begin{array}{r}\text { Préjudice } \\
\text { (pécuniaire) }\end{array}$ & $\begin{array}{l}\text { corporel } \\
\text { (non } \\
\text { pécuniaire) }\end{array}$ \\
\hline & & \\
\hline
\end{tabular}

L'idée d'une troisième catégorie n'était pas absente du Code civil du Bas Canada ${ }^{9}$. Néanmoins, le défaut du législateur de proposer une classification

8. Carbonnier, supra, note 2, $\mathrm{n}^{\text {os }}$ 206-207; Malinvaud, supra, note 2, $\mathrm{n}^{\circ}$ 211; Mazeaud, Leçons, supra, note $2, \mathrm{n}^{\circ} 409$.

Il faut noter que le professeur Tancelin, dans la plus récente édition de son ouvrage, propose une interprétation différente de la notion de préjudice corporel :

$\ll[\mathrm{L}]$ a distinction entre préjudice matériel et préjudice moral est insuffisante à rendre compte des solutions du droit positif. C'est pourquoi on distingue plus volontiers aujourd'hui le préjudice à la personne, dit "préjudice corporel" [...], et le préjudice aux biens, dit préjudice "matériel". L'un comme l'autre peut être soit matériel, soit moral.»

M. Tancelin, supra, note $2, \mathrm{n}^{\circ} 556$.

9. Ainsi, dans le droit antérieur, était-il nécessaire d'identifier les «lésions ou blessures corporelles» afin de déterminer le délai de prescription applicable à la réclamation de la victime selon l'art. 2262 par. 2 C.c.B.C. De même, l'identification de «blessures corporelles» était essentielle pour justifier l'application de l'article $1056 \mathrm{~b}$ al. 4 C.c.B.C. Plus récemment, l'art. $2260 \mathrm{a}$ C.c.B.C. faisait référence au préjudice «corporel ou mental» causé à un patient en matière de responsabilité médicale ou hospitalière. 
(1993) 24 Rréjudice matériel, corporel et moral :

(1993) 24 R.D.U.S. variations sur la classification tripartite du préjudice

systématique a eu pour conséquence de laisser aux tribunaux et à la doctrine le soin d'élaborer cette classification tripartite.

Les développements majeurs survenus dans la jurisprudence canadienne en matière d'indemnisation du préjudice corporel ont permis de saisir la spécificité de ce type de préjudice. Pourtant, dans nombre de décisions récentes, les tribunaux évitaient toujours les problèmes liés au choix de l'une ou l'autre des classifications proposées, référant simplement aux chefs de réclamation (pertes de profit, inconvénients, atteinte à la dignité, souffrances, etc.) sans les rattacher à une catégorie particulière de préjudice.

De la même façon, la doctrine récente ne tranchait pas clairement en faveur de l'un ou l'autre des modes de classification : certains auteurs référaient encore à la classification bipartite ${ }^{10}$ alors que d'autres adhéraient à la classification tripartite ${ }^{11}$.

Il faut toutefois concéder que le défaut de consacrer un mode particulier de classification n'emportait pas de conséquences significatives, puisque le législateur lui-même n'en proposait aucun et, surtout, que peu de règles étaient conditionnées par le rattachement d'un chef de dommage à l'une ou l'autre des catégories de préjudice. Or, la réforme du droit québécois modifie les règles du jeu. En effet, le législateur exprime clairement sa préférence pour la classification tripartite du préjudice (art. 1457, 1458 et 1607 C.c.Q.) ${ }^{12}$. Plus encore, en multipliant les règles propres à l'une ou l'autre des catégories de

10. En droit québécois : J. Pineau et D. Burman, Théorie des obligations, $2^{\mathrm{e}}$ éd., Montréal, Thémis, 1988, $\mathrm{n}^{\text {os }} 347-350$. En droit français : Larroumet, supra, note 2, $\mathrm{n}^{\text {os }}$ 649-653; Le Tourneau, supra, note 2, $\mathrm{n}^{\circ}$ 562; Malaurie et Aynès, supra, note 2, $\mathrm{n}^{\text {os }}$ 241-247; Viney, supra, note 2, $\mathrm{n}^{\text {os }} 250$ et s. Voir aussi Marty et Raynaud, supra, note 2, $\mathrm{n}^{\text {os }} 430-432$; Starck, supra, note $2, \mathrm{n}^{\text {os }} 98$ et 106.

11. En droit québécois : J.-L. Baudouin, La responsabilité civile délictuelle, $3^{\mathrm{e}}$ éd., Cowansville, Yvon Blais, 1990, passim. En droit français : Carbonnier, supra, note 2, n ${ }^{\text {os }} 206$ et 207; Malinvaud, supra, note 2, $\mathrm{n}^{\circ}$ 211; Mazeaud, Leçons, supra, note 2, $\mathrm{n}^{\circ} 409$. Voir aussi J. Flour et J.-L. Aubert, Droit civil. Les obligations, vol. 2, Le fait juridique, $5^{\mathrm{e}}$ éd., Paris, Armand Colin, 1991, ${ }^{\circ} 135$; Weill et Terré, supra, note 6, $\mathrm{n}^{\text {os }}$ 606-612.

12. Les premiers projets de réforme allaient plutôt vers une classification bipartite du préjudice : Office de révision du Code civil, Rapport sur le Code civil du Québec, vol. I, Projet de Code civil, Québec, Éditeur officiel, 1978, livre V, art. 293 («Le préjudice matériel ou moral est susceptible de réparation.»). Voir aussi Rapport sur le Code civil, vol. II, Commentaires, t. 2, Québec, Éditeur officiel, 1978, p. 686. Le développement de règles spécifiques à l'indemnisation du préjudice corporel a certainement contribué à ce revirement. D'ailleurs, on retrouvait déjà, dans ce projet de réforme, des dispositions référant expressément aux «blessures corporelles» (livre V, art. 292, 296), à l'«atteinte à l'intégrité physique» (livre V, art. 297) et à l'«atteinte à la personne» (livre V, art. 301).

Déjà, au stade de l'avant-projet de loi, la classification tripartite était avancée par le législateur: Assemblée Nationale, Loi portant réforme au Code civil du Québec du droit des obligations, $1^{\text {re }}$ sess., $33^{\mathrm{e}}$ lég., art. 1515 al. 2 et 1659. 
préjudice, il donne un rôle significatif à la classification retenue et impose, par le fait même, une plus grande rigueur terminologique.

\section{Impacts de la classification du préjudice dans le nouveau droit de la responsabilité}

Diverses dispositions du Code civil du Québec illustrent l'importance qu'accorde désormais le législateur à la classification du préjudice.

Certains articles font référence aux trois catégories de façon générale, sans pour autant attribuer de conséquences particulières à l'une ou l'autre d'entre elles. Il en va ainsi notamment des articles de base de la responsabilité civile extracontractuelle et contractuelle (art. 1457 et $1458^{13}$ C.c.Q.) et de l'indemnisation du préjudice (art. 1607 C.c.Q.) ${ }^{14}$. Plus intéressantes pour les fins de notre propos, toutefois, sont les dispositions en vertu desquelles le rattachement d'un chef de réclamation à une catégorie particulière de préjudice s'avère déterminant. En effet, le législateur a cherché, dans plusieurs cas, à offrir une protection particulière à la victime d'un préjudice corporel et, à l'occasion, d'un préjudice moral.

Ainsi, en matière de régimes matrimoniaux, le législateur déclare que le droit à des dommages-intérêts et l'indemnité reçue en réparation d'un préjudice corporel ou moral sont des biens propres à l'époux victime de ce préjudice (art. 454 C.c.Q.). Cette disposition exprime bien la volonté du législateur de systématiser l'emploi de la classification tripartite du préjudice, puisque la disposition correspondante dans l'ancien Code civil du Québec

13. Il aurait pu en être autrement de cette disposition. Initialement, le législateur était tenté de soustraire la victime d'un préjudice corporel au principe selon lequel toute action fondée sur l'inexécution d'une obligation contractuelle doit nécessairement relever de la responsabilité contractuelle. La première version de l'art. 1458 C.c.Q. se lisait ainsi :

«Toute personne a le devoir d'honorer les engagements qu'elle a contractés.

Elle est, lorsqu'elle manque à ce devoir, responsable du préjudice, corporel, moral ou matériel, qu'elle cause à son cocontractant et tenue de réparer ce préjudice; ni elle ni le cocontractant ne peuvent alors se soustraire à l'application des règles du régime contractuel de responsabilité pour opter en faveur de règles qui leur seraient plus profitables, mais si le préjudice est corporel, seules les règles du régime extracontractuel de responsabilité s'appliquent.» [Nos italiques.]

Assemblée Nationale, Projet de loi 125, Code civil du Québec, $1^{\text {re }}$ sess., $34^{\mathrm{e}}$ lég., présentation, art. 1454. Le législateur s'est toutefois ravisé par un amendement qui supprimait ce dernier passage; à ce sujet, voir Assemblée Nationale, Journal des débats. Commissions parlementaires. Sous-commission des institutions, $1^{\mathrm{re}}$ sess., $34^{\mathrm{e}}$ lég., 19 sept. 1991, p. SCI-513-SCI-515 et 9 oct. 1991, p. SCI-569-SCI-570.

14. Un autre cas où le législateur réfère spécifiquement aux trois catégories de préjudice sans attacher de conséquences particulières à l'une d'entre elles est l'art. 2926 C.c.Q., relatif au point de départ de la prescription en cas de manifestation graduelle ou tardive du préjudice. 
(1993) Préjudice matériel, corporel et moral :

(1993) 24 R.D.U.S. variations sur la classification tripartite du préjudice

référait plutôt au «droit à des dommages-intérêts et l'indemnité reçue pour atteinte à la personne» (art. 486 C.c.Q. ancien).

Un changement particulièrement important apporté par la réforme concerne les clauses d'exclusion et de limitation de responsabilité. Constatant que la victime d'un préjudice corporel ou moral méritait une plus grande protection, en raison de la «primauté de la personne humaine» reconnue dans le Code $^{15}$, le législateur invalide toute convention visant à limiter ou exclure la responsabilité eu égard à ces deux catégories de préjudice. Désormais, de telles clauses n'affectent la responsabilité que pour le seul préjudice matériel.

Dans le même esprit, le législateur a repris et étendu la protection de la victime en matière de quittances, transactions et déclarations obtenues dans les jours suivant la survenance du préjudice (art. 1056b C.c.B.C.). Parmi les changements intervenus dans le cadre de la réforme sur cette question ${ }^{16}$, il faut souligner que cette protection n'est plus limitée à la seule victime d'un préjudice corporel et s'étend désormais à la victime d'un préjudice moral (art. 1609 C.c.Q.). Les légistes sont toutefois silencieux sur les motifs à l'origine de cette modification, laquelle résulte d'ailleurs d'un amendement introduit après la présentation du projet de loi $125^{17}$.

En matière d'indemnisation du préjudice, une série d'articles se rapporte spécifiquement au préjudice corporel (art. 1614 à 1616 C.c.Q.). Ces dispositions de droit nouveau visent, chacune à leur façon, à protéger la victime d'un

15. Commentaires du ministre de la Justice, t. I, Québec, Publications du Québec, 1993, p. 903 (sous l'art. 1474 C.c.Q.).

Cette idée n'est pas nouvelle; voir notamment R. Nerson, Droits extrapatrimoniaux, Paris, Librairie générale de Droit et de Jurisprudence, 1939, nº 187, p. 413; Y. Lambert-Fâ̂vre, De la responsabilité encourue envers les personnes autres que la victime initiale. Le problème dit du «dommage par ricochet», thèse de doctorat en droit, Université de Lyon, 1959 [non publiée], p. 280.

16. Sur l'ensemble des modifications apportées au régime de l'art. 1056b C.c.B.C., voir C. Masse, «La responsabilité civile», dans Barreau du Québec et Chambre des notaires du Québec, dir., La réforme du Code civil, t. II, Québec, Presses de l'Université Laval, 235, nº 105.

17. Une version préliminaire des commentaires se lisait ainsi :

«[Cette modification] apporte une précision qui a paru s'imposer, en raison du lien étroit qui existe normalement entre le préjudice moral et le préjudice corporel.»

Gouvernement du Québec, Ministère de la Justice, Projet de loi 125. Code civil du Québec. Commentaires détaillés sur les dispositions du projet, Amendements, vol. 2, Québec, Gouvernement du Québec, Ministère de la Justice, 1991.

Ce commentaire paraphrasait, en fait, des propos émis dans le cadre des travaux de la souscommission des Institutions : Journal des débats, supra, note 13, 9 oct. 1991, p. SCI-544SCI-545. Les travaux de la Sous-commission font état de la difficulté de distinguer le préjudice corporel et le préjudice moral. Sur la confusion existant au sujet de la distinction du préjudice corporel (principalement dans sa dimension non pécuniaire) et du préjudice moral, voir ci-dessous, partie III.A. 
préjudice corporel ${ }^{18}$. Tout d'abord, le législateur allège la tâche de la victime de préjudice corporel quant à la preuve du taux d'actualisation applicable à sa réclamation (art. 1614 C.c.Q.) ${ }^{19}$. De même, le tribunal peut réserver le droit de la victime de demander, dans les trois ans, des dommages-intérêts supplémentaires en réparation du préjudice corporel, lorsque l'étendue de ce préjudice ne saurait être établie avec une précision suffisante (art. 1615 C.c.Q. ${ }^{20}$. Enfin, le mineur victime d'un préjudice corporel peut profiter d'une exception au principe de l'attribution forfaitaire de l'indemnisation et se faire octroyer par le tribunal des dommages-intérêts sous forme de rente ou de versements périodiques (art. 1616 C.c.Q.) ${ }^{21}$.

Par ailleurs, la réclamation de la victime d'un préjudice corporel ne peut désormais être soumise à un délai de prescription inférieur à trois ans, ni par une intervention directe du législateur réduisant le délai applicable, ni de façon indirecte par l'imposition d'un avis préalable à la poursuite (art. 2930 C.c.Q.). Il s'agit là d'une innovation majeure, notamment en matière de responsabilité municipale.

Ces différentes dispositions, majoritairement issues de la réforme, démontrent l'importance accrue de la classification du préjudice dans le droit de la responsabilité. Si les tribunaux ont généralement intérêt à invoquer explicitement la classification retenue par le législateur, cet exercice sera tout simplement indispensable dans les cas où le rattachement d'un chef de réclamation (perte de revenus, coût des soins, souffrances, inconvénients, etc.) à l'une ou l'autre des catégories entraîne l'application d'une règle particulière. De façon générale, cet effort de systématisation devrait se faire assez aisément. Il faut toutefois s'arrêter à certaines difficultés auxquelles les juristes pourraient être confrontés.

18. La question ne se limite alors pas à l'identification de ce qui constitue un préjudice corporel, puisqu'il faut également déterminer quelles sont les personnes qui peuvent s'en prétendre victimes. Sur le statut de la victime médiate d'une atteinte à l'intégrité corporelle, voir cidessous, partie III.B.

19. On pourrait certes prétendre que cette protection est toute relative, puisqu'on empêche du même coup la victime de faire la preuve d'un taux d'actualisation plus favorable que celui établi par règlement. Néanmoins, le législateur a probablement jugé - à tort ou à raison -que le bénéfice possible en faisant la preuve d'un taux d'actualisation plus favorable serait contrebalancé par le coût afférant à la présentation de cette preuve : Commentaires du ministre de la Justice, supra, note 15, p. 998-999 (sous l'art. 1614 C.c.Q.).

20. Cette disposition est complétée par les art. 26.1, 71.1, 469.1, 510.1 et 762 al. 2 par. b) C.p.c.

21. Dans tous les autres cas, le tribunal est limité à l'octroi d'une somme forfaitaire; seule une entente entre les parties peut permettre de faire échec au principe énoncé au premier alinéa de l'art. 1616 C.c.Q.

Les commentaires des légistes font état d'une volonté, dans le cadre de cette disposition, de protéger à la fois les intérêts du créancier mineur et ceux du débiteur : Commentaires $d u$ ministre de la Justice, supra, note 15, p. 1001 (sous l'art. 1609 C.c.Q.); voir aussi Journal des débats, supra, note 13, 9 oct. 1991, p. SCI-547-SCI-549. 
(1993) 24 R Péjudice matériel, corporel et moral :

(1993) 24 R.D.U.S. variations sur la classification tripartite du préjudice

\section{Difficultés possibles en matière de classification du préjudice}

On peut anticiper certaines difficultés dans l'application du mode de classification retenu par le législateur. Ainsi, il semble de mise de s'interroger sur les différences de traitement entre le préjudice corporel et le préjudice moral (A). De même, on peut soulever la question de la nature du préjudice subi par la victime médiate (ou «par ricochet») d'une atteinte à l'intégrité corporelle (B). Enfin, on ne saurait ignorer les problèmes liés à l'harmonisation des dispositions du Code avec la législation en vigueur (C).

\section{A. Préjudice corporel et préjudice moral : double standard ou confusion des termes?}

On conçoit aisément que le législateur n'ait pas cherché à accorder de protection particulière à la victime d'un préjudice matériel. Inversement, il est facile d'expliquer son souci d'assurer une telle protection à la victime d'un préjudice corporel, quand on connaît l'importance qu'accorde le législateur à la sauvegarde de l'intégrité de la personne.

Le statut du préjudice moral, dans cette optique, suscite certaines interrogations. Comment le législateur entrevoit-il cette forme de préjudice? La réponse est difficile à saisir, du moins si l'on en juge par la protection accordée à la victime d'un préjudice moral - plus étendue que celle octroyée à la victime d'un préjudice matériel, mais plus limitée que celle dont jouit la victime d'un préjudice corporel.

Une première position possible serait de soutenir que, pour le législateur, le préjudice corporel touche la personne de façon plus fondamentale que le préjudice moral. Mais comment, alors, expliquer que certaines dispositions accordent malgré tout la même protection pour les deux types de préjudice (art. 1474, 1609 C.c.Q.)? On pourrait soulever la possibilité que le législateur ait erronément étendu de telles formes de protection à la victime d'un préjudice moral $^{22}$. En effet, il ne serait pas surprenant que le préjudice moral (désormais limité, dans le cadre de la classification tripartite, aux situations où le préjudice non pécuniaire survient indépendamment d'une atteinte à l'intégrité corporelle) soit, à l'occasion, confondu avec le préjudice corporel entendu dans sa dimension non pécuniaire ${ }^{23}$. Ainsi, dans les commentaires des légistes, fait-on référence au préjudice moral en ce qui concerne le préjudice esthétique ${ }^{24}$, un

22. Cette hypothèse pourrait être confirmée du fait que l'art. 1609 C.c.Q., dans sa version originale, se limitait au seul préjudice corporel, de la même façon que l'art. 1056b C.c.B.C. se limitait aux cas de blessures corporelles.

23. À ce sujet, voir les commentaires supra, note 17.

24. $\quad$ Commentaires du ministre de la Justice, supra, note 15, p. 993 (sous l'art. 1607 C.c.Q.). 
chef de réclamation se rattachant plutôt à la catégorie du préjudice corporel. D'ailleurs, la difficulté de distinguer le préjudice corporel et le préjudice moral a été évoquée dans le cadre des travaux de la sous-commission des Institutions, lors de la discussion de l'amendement ayant pour but d'étendre le domaine de l'article 1609 C.c.Q. au préjudice moral ${ }^{25}$.

Si l'on considère, au contraire, que le législateur conçoit le préjudice corporel et le préjudice moral comme méritant la même forme de protection, il faut alors trouver une justification aux règles s'appliquant uniquement à la victime d'un préjudice corporel. Cette explication vient plus aisément pour les articles consacrés à l'indemnisation du préjudice corporel (art. 1614 à 1616 C.c.Q.). En effet, le préjudice corporel (principalement dans sa dimension pécuniaire) est le plus susceptible de variations dans le futur, d'où l'importance de prévoir des mécanismes tels la détermination du taux d'actualisation ou la possibilité de réclamer des dommages-intérêts additionnels. Quant à la possibilité d'ordonner le paiement de dommages-intérêts sous forme de rente ou de versements périodiques, elle se justifie par les sommes élevées auxquelles le créancier victime d'un préjudice corporel peut avoir droit - sommes qui sont souvent largement supérieures à celles octroyées en réparation d'un préjudice moral. La seule disposition où il s'avère plus difficile de fournir une explication pratique de la différence de traitement est celle relative au délai de prescription minimal applicable à un recours en responsabilité (art. 2930 C.c.Q.). Pourquoi ne pas avoir fixé le délai minimal de trois ans pour le préjudice moral comme pour le préjudice corporel? Il pourrait certes s'agir d'un oubli. Néanmoins, le maintien du délai de prescription d'un an en cas d'atteinte à la réputation recours destiné principalement, et souvent exclusivement, à la réparation du préjudice moral -pourrait démontrer que le législateur agissait alors en toute connaissance de cause.

De fait, l'élément le plus inquiétant n'est pas tant la différence de traitement entre le préjudice corporel et le préjudice moral, mais plutôt la confusion qui semble subsister chez certains juristes quant au domaine respectif de l'un et de l'autre. En ce sens, il est souhaitable que les autorités s'efforcent, à l'avenir, de donner l'heure juste à cet égard ${ }^{26}$.

25. Journal des débats, supra, note 13, 9 oct. 1991, p. SCI-545.

26. Un arrêt récent de la Cour d'appel montre d'ailleurs des signes encourageants dans une optique de clarification. Dans Gagnon c. Béchard, [1993] R.J.Q. 2019 (C.A.), la Cour d'appel prend soin de distinguer le préjudice découlant de «lésions ou blessures corporelles» du préjudice moral. La Cour d'appel en vient à la conclusion que le préjudice allégué dans une affaire d'attouchements sexuels n'est pas nécessairement lié à des lésions ou blessures corporelles et, par conséquent, que la courte prescription de l'art. 2262 C.c.B.C. ne trouve pas application. Si ce résultat peut surprendre, il faut préciser qu'il s'agit en l'espèce d'une requête en irrecevabilité, et que l'arrêt se fonde exclusivement sur les faits allégués, lesquels ne font aucune mention d'atteintes d'ordre corporel. 
(1993) Préjudice matériel, corporel et moral :

(1993) 24 R.D.U.S. variations sur la classification tripartite du préjudice

dans le nouveau droit de la responsabilité

S'il s'avère important en pratique de déterminer ce qui constitue un préjudice corporel (pour bien le distinguer du préjudice moral), il est tout aussi pertinent de s'interroger sur l'identité de la personne qui s'en dit victime. Ceci nous amène à traiter de la nature du préjudice subi par la victime médiate d'une atteinte à l'intégrité corporelle.

\section{B. La victime médiate d'une atteinte à l'intégrité corporelle subit-elle un préjudice «corporel»?}

Parmi les problèmes potentiels liés à la classification du préjudice, l'un des plus importants concerne la victime d'un préjudice «par ricochet» ou «réfléchi», c'est-à-dire la victime «médiate». Quelle est la nature du préjudice subi par cette personne, lorsque la victime immédiate subit un préjudice corporel $^{27}$ ? Doit-on qualifier le préjudice réfléchi de préjudice corporel (dans ses dimensions pécuniaire et non pécuniaire) ou de préjudice matériel et moral? Cette question se posera sans doute plus fréquemment dans l'avenir, en raison de la multiplication des dispositions dont l'application est réservée au préjudice corporel ou moral.

Ainsi, on peut se demander si le tiers peut bénéficier des dispositions particulières à l'indemnisation du préjudice corporel, notamment quant à l'établissement du taux d'actualisation et du droit de demander des dommagesintérêts supplémentaires (art. 1614 et 1615 C.c.Q.). De même, il devient pertinent de s'interroger sur l'applicabilité des clauses d'exclusion ou de limitation de responsabilité, dans les cas où la victime médiate se situe dans le cercle contractuel (art. 1474 C.c.Q. ${ }^{28}$. Enfin, la victime médiate ne saurait se prémunir contre un délai de prescription inférieur à trois ans ou l'imposition d'un avis préalable au même effet si le préjudice subi est uniquement considéré comme matériel et moral (art. 2930 C.c.Q. a contrario).

Ce problème de qualification n'était pas entièrement inconnu dans le droit antérieur. En effet, une question semblable s'est posée au sujet de l'article 2262 par. 2 C.c.B.C., lequel imposait un délai de prescription d'un an en matière de «lésions ou blessures corporelles». Les tribunaux avaient été amenés à

27. Un problème similaire se poserait pour la victime d'un préjudice corporel demandant réparation à une personne dont les agissements l'ont privée d'un recours utile contre l'auteur de l'atteinte à son intégrité corporelle - par exemple, un avocat ayant laissé filer la prescription. Ici, toutefois, l'identité de la victime demeure la même et c'est plutôt l'identité de l'auteur de l'atteinte (et du fondement du recours) qui varie. La solution retenue ne serait donc pas nécessairement la même.

28. Ce pourrait être le cas en présence d'un contrat intervenu à la fois avec la victime immédiate et la victime médiate, mais aussi dans les cas où la victime médiate bénéficie d'une stipulation pour autrui dans le cadre d'un contrat intervenu entre la victime immédiate et le cocontractant responsable du préjudice. 
décider si la victime médiate était soumise au même délai de prescription que la victime immédiate, ou si elle bénéficiait du délai de deux ans de l'article 2261 C.c.B.C. en matière de délits et quasi-délits.

La réponse est venue dans l'arrêt Regent Taxi, rendu par le Conseil privé. Dans cette affaire, la victime médiate avait intenté son recours plus d'un an mais moins de deux ans après la survenance du préjudice à la victime immédiate. La Cour d'appel et la Cour suprême avaient approuvé les motifs du juge de première instance à l'effet que le recours de la victime médiate n'était pas prescrit puisqu'il ne s'agissait pas - pour reprendre l'article 2262 C.c.B.C. tel qu'il se lisait à l'époque - d'«injures corporelles» ${ }^{29}$. Le Conseil Privé en a jugé autrement. Trois arguments étaient avancés au soutien de cette position. Les deux premiers découlent de l'interprétation de l'article 2262 C.c.B.C. D'abord, Lord Russell of Killowen affirme que les mots «for bodily injuries» ne devaient pas être interprétés littéralement, puisqu'on ne réfère pas à un recours pour préjudice corporel mais plutôt pour préjudice consécutif à une atteinte corporelle («damages sustained from the wrongful infliction of bodily injuries») $)^{30}$. Reconnaissant que ce premier argument n'est pas à lui seul convaincant, il en ajoute un second : l'article 2242 C.c.B.C. fait référence à l'article 1056 C.c.B.C., disposition qui concerne manifestement le recours de victimes médiates ${ }^{31}$. Le dernier argument avancé par le Conseil privé repose principalement sur des considérations d'équité :

«Indeed, it would be strange if it were otherwise; for the result then would be $[\ldots .$.$] that in the case of a wrongful infliction of bodily$ injuries, the physical victim must sue within one year while third parties may take twice as long before asserting their claims. Their Lordships find it impossible to suggest any plausible reason why this should be so. If any difference in the periods of limitation should be made between the two plaintiffs, they would have expected to find the respective positions reversed $\gg^{32}$. [Nos italiques.]

Doit-on considérer que la position exprimée dans Regent Taxi répond à la question de façon claire et définitive?

Cette solution ne va pas nécessairement de soi. En effet, à première vue, la logique commande que l'on associe le qualificatif de préjudice corporel à la

29. Regent Taxi \& Transport Co. c. Congrégation des Petits Frères de Marie, dits Frères Maristes, (1928) 46 B.R. 96, p. 97-98 et 108-109, conf. par [1929] R.C.S. 650, p. 673-674 et 710 .

30. Regent Taxi and Transport Co.c. Congrégation des Petits Frères de Marie, (1932) 53 B.R. 157 (P.C.), p. 163. Voir aussi Baudouin, supra, note 11, $\mathrm{n}^{\circ} 1109$.

31. Ibid., p. 163 .

32. Ibid., p. 163-164. 
(1993) 24 Rréjudice matériel, corporel et moral :

(1993) 24 R.D.U.S. variations sur la classification tripartite du préjudice

personne victime de l'atteinte à l'intégrité corporelle, puisque c'est cette atteinte qui caractérise la catégorie du préjudice corporel. Or, si des tiers souffrent par la même occasion, ce n'est pas tant en raison de cette atteinte que du préjudice subi par la victime immédiate. D'ailleurs, en droit français -où le rattachement à l'une ou l'autre des catégories, il est vrai, emporte peu de conséquences - les auteurs qui adhèrent à la classification tripartite du préjudice ont généralement tendance à qualifier le préjudice subi par la victime médiate de préjudice matériel (perte du soutien financier provenant de la victime immédiate, dépenses encourues au bénéfice de la victime immédiate, etc.) et moral (perte de soutien affectif, perte de jouissance, etc. $)^{33}$.

En modifiant les enjeux du débat, la réforme nous amène du même coup à réévaluer la position adoptée par le Conseil privé. En effet, il ne faut pas oublier que la solution proposée dans Regent Taxi reposait en partie sur l'interprétation de dispositions aujourd'hui disparues. Quant à l'argument d'équité retenu dans cet arrêt - soit de chercher à éviter que la victime immédiate ne soit soumise à des règles plus sévères que celles applicables à la victime médiate le problème ne se pose plus. En effet, si l'on considère les règles nouvelles abordées plus haut, force est de constater que le tiers ne pourrait désormais être traité de façon plus favorable que la victime immédiate du simple fait que l'on qualifie différemment le préjudice subi par l'une et l'autre des victimes ${ }^{34}$. Pour reprendre la formule employée par Lord Russell of Killowen, les positions respectives sont inversées : si la qualification du préjudice peut désavantager une victime, ce ne saurait être la victime immédiate de l'atteinte à l'intégrité corporelle. Il est d'ailleurs surprenant que cette distinction n'ait pas été soulevée dans l'arrêt Lapointe c. Hôpital Le Gardeur ${ }^{35}$, où la Cour d'appel a appliqué le raisonnement tiré de Regent Taxi ${ }^{36}$. Dans cet arrêt, contrairement à d'autres affaires où la solution proposée par le Conseil privé a été appliquée à la même

33. Carbonnier, supra, note $2, \mathrm{n}^{\circ}$ 207; Malinvaud, supra, note $2, \mathrm{n}^{\circ} 212$. Voir aussi Weill et Terré, supra, note $11, \mathrm{n}^{\mathrm{os}}$ 610-612. De façon générale, sur le recours de la victime médiate en matière d'atteinte à l'intégrité corporelle, voir D. Gardner, L'évaluation du préjudice corporel, Cowansville, Yvon Blais, 1994, $\mathrm{n}^{\text {os }} 344$ et s.; J. Dupichot, Des préjudices réfléchis nés de l'atteinte à la vie ou à l'intégrité corporelle, Paris, Librairie générale de Droit et de Jurisprudence, 1969; M. Le Roy, L'évaluation du préjudice corporel, $9^{\mathrm{e}}$ éd., Paris, Litec, $1982, \mathrm{n}^{\text {os }} 108$ et s. Voir aussi Lambert-Faîvre, supra, note 15.

34. Cette affirmation est vraie, à tout le moins, quant aux dispositions spécifiques à la nature du préjudice subi.

Si l'on envisage plutôt les conditions de la responsabilité, il pourrait arriver que le régime applicable à la victime médiate s'avère plus avantageux que celui auquel est soumise la victime, notamment en raison de l'interdiction de l'option de régime.

35. [1989] R.J.Q. 2619 (C.A.), infirmé pour d'autres motifs par [1992] 1 R.C.S. 363.

36. Ibid., p. 2626-2627. 
disposition (art. 2262 C.c.B.C.) ${ }^{37}$, l'article sous étude (art. 2260a C.c.B.C.) plaçait la victime médiate dans une position identique à celle de la victime immédiate ${ }^{38}$.

Il est vrai néanmoins que le refus de qualifier de corporel le préjudice subi par la victime médiate serait de nature à désavantager cette dernière dans certaines circonstances. Doit-on, par souci d'équité, accorder à cette victime une protection aussi étendue que celle dont bénéficie la victime immédiate? L'hypothèse d'un traitement moins favorable n'est pas particulièrement choquante si le tiers n'entretient pas de liens étroits avec la victime immédiate par exemple, l'employeur privé des services de son salarié. Il en va autrement des proches - conjoint ou parents - qui encourent des déboursés importants pour le mieux-être de la victime immédiate. Peut-être pourrait-on aller plus loin et considérer que la proximité requise pour donner droit à l'indemnisation du préjudice réfléchi suffit pour lier ce préjudice à l'atteinte corporelle ${ }^{39}$. Dès lors, on serait tenté de suivre la voie tracée par le Conseil privé dans Regent Taxi et qualifier de corporel le préjudice subi par la victime médiate ${ }^{40}$. Il serait néanmoins souhaitable que les tribunaux, quelle que soit la solution retenue, cherchent à articuler cette solution en considérant l'état du droit actuel, et qu'ils se gardent d'appliquer mécaniquement une solution élaborée dans un tout autre contexte.

37. J. \& A. Levasseur Construction Inc. c. Tremblay Express Ltée, [1978] C.A. 249, p. 250-251; Comtois Méconse c. Hooper, [1987] R.R.A. 819, p. 827 (C.S.). Voir aussi Hôpital NotreDame c. Patry, [1975] 2 R.C.S. 388; Centre hospitalier St-Vincent-de-Paul c. Côté, [1981] C.S. 300.

38. Voir contra, Centre hospitalier St-Vincent-de-Paul c. Côté, ibid., p. 302; Comtois Méconse c. Hooper, ibid., p. 827. Dans ces deux décisions, le tribunal énonce que le délai de trois ans de l'art. 2260a C.c.B.C. est réservé au patient, mais soumet la victime médiate à la prescription annale de l'art. 2262 C.c.B.C. (lequel ne serait pas limité à la seule victime des lésions et blessures corporelles).

39. Le professeur Gardner souligne la tendance des tribunaux à considérer que le recours appartient alors à la victime immédiate qui, à son tour, indemnise le proche concerné : Gardner, supra, note $33, \mathrm{n}^{\circ} 356$.

40. Le professeur Baudouin suggère implicitement une telle solution en affirmant que, suite à la disparition de l'art. 1056 C.c.B.C., les réclamations contractuelles en cas de décès seraient soumises aux règles propres au préjudice corporel : J.-L. Baudouin, Les obligations, $4^{\mathrm{e}}$ éd., Cowansville, Yvon Blais, 1993, $\mathrm{n}^{\mathrm{o}} 776$. 
(1993) 24 Rréjudice matériel, corporel et moral :

(1993) 24 R.D.U.S. variations sur la classification tripartite du préjudice

dans le nouveau droit de la responsabilité

\section{Classification tripartite et législation québécoise}

Par une reconnaissance formelle de la classification tripartite du préjudice et, surtout, par les conséquences non négligeables qu'entraîne le rattachement d'un chef de réclamation à une catégorie particulière de préjudice, le législateur appelle une utilisation plus fréquente et systématique de cette classification. Il attend donc des tribunaux, chargés d'appliquer les règles de droit nouveau, une plus grande rigueur terminologique. C'est la même rigueur que l'on devrait attendre du législateur lui-même dans la désignation des catégories de préjudice. Pour en juger, il convient donc de se pencher sur la pratique législative en matière de désignation du préjudice.

Dans la législation québécoise, il arrive que certaines dispositions ne respectent pas le mode de classification du préjudice proposé par le Code civil. Ainsi, il faut regretter le défaut du législateur de modifier les articles faisant spécifiquement appel à la classification bipartite du préjudice. Le cas le plus flagrant est l'article 49 alinéa 1 de la Charte des droits et libertés de la personne ${ }^{41}$, qui énonce qu'une atteinte «confère à la victime le droit d'obtenir la cessation de cette atteinte et la réparation du préjudice moral ou matériel qui en résulte.» Une modification législative serait la bienvenue, afin de dissiper toute confusion sur l'étendue de la réparation octroyée en vertu de la Charte. De la même façon, le législateur utilise à l'occasion des expressions telles que le «dommage aux biens» plutôt que les expressions consacrées dans la classification tripartite ${ }^{42}$.

Bien entendu, il se trouve certaines situations où une appellation particulière du préjudice est justifiée par l'objet de la loi. Ainsi, dans le cadre de la Loi sur l'assurance automobile $e^{43}$, le législateur donne une portée différente aux expressions «dommage corporel» ${ }^{44}$ et «dommage matériel» ${ }^{45}$. Il fait de même dans le cadre de la Loi sur la protection de la santé publique au sujet du «dommage corporel» ${ }^{46}$. Une telle dérogation n'est pas répréhensible, à tout le moins lorsque le législateur prend la peine de recourir à une définition pour exprimer sa conception particulière de l'une ou l'autre des catégories de préjudice.

\footnotetext{
41. L.R.Q. c. C-12.

42. Par exemple, Loi sur l'aide et l'indemnisation des victimes d'actes criminels, L.Q. 1993, c. 54 , art. $9\left(2^{\circ}\right)$

43. L.R.Q. c. A-25.

44. Ibid., art. 2, «préjudice corporel». Le législateur donne alors un sens quelque peu élargi au préjudice corporel, puisqu'il y inclut les «dommages aux vêtements que porte la victime». 45. Ibid., art. 24.1, al. 1.

46. L.R.Q. c. P-35, art. 16.1 b) : «dommage permanent grave, physique ou mental, incluant le décès». [Nos italiques.]
} 
Par ailleurs, on ne peut nier l'incidence de certaines dispositions législatives lorsqu'il s'agit de définir chacune des catégories de préjudice comprises dans la classification tripartite. L'exemple le plus frappant est certes la définition du préjudice corporel. En effet, dans de nombreuses dispositions, le législateur précise que ce préjudice inclut non seulement les atteintes à l'intégrité physique, mais également à l'intégrité $p s y c h i q u e^{47}$. Pour le législateur, le préjudice corporel devrait désormais se concevoir comme toute atteinte au droit à l'inviolabilité de la personne, considéré de façon globale, sans se limiter aux aspects strictement physiologiques. Dans cette optique, le préjudice psychique s'intégrerait à la catégorie du préjudice corporel - plutôt que de former une catégorie distincte de préjudice ${ }^{48}$ - et, il faut en convenir, rendrait plus complexe encore la distinction entre le préjudice corporel et le préjudice moral.

\section{Conclusion}

De nombreuses critiques ont été adressées au législateur eu égard à la rédaction du nouveau code civil. Pourtant, il ne faudrait pas perdre de vue les efforts considérables faits par le législateur en vue d'améliorer la terminologie juridique. Par exemple, l'utilisation du terme «préjudice», de préférence à celui de «dommage», marque mieux la distinction entre les conditions et les effets de la responsabilité ${ }^{49}$.

De la même façon, la reconnaissance formelle de la classification tripartite constitue un effort louable en ce sens. S'il est vrai que certaines clarifications s'imposent encore - tant de la part des tribunaux que du législateur -, il s'agit tout au plus de retouches destinées à mettre en valeur le travail accompli.

47. Loi sur l'aide et l'indemnisation des victimes d'actes criminels, supra, note 42 , art. $9\left(1^{\circ}\right)$; Loi visant à favoriser le civisme, L.R.Q. c. C-20, art. 1 d), mod. par la Loi sur l'aide et l'indemnisation des victimes d'actes criminels, ibid., art. $197\left(3^{\circ}\right)$; Loi sur les accidents $d u$ travail et les maladies professionnelles, L.R.Q. c. A-3.001, art. 83; Loi sur l'assurance automobile, L.R.Q. c. A-25, art. 2. Voir aussi la Loi sur la protection de la santé publique, ibid., art. $16.1 \mathrm{~b}$ ).

48. En ce sens, il est regrettable que le législateur mentionne le «préjudice corporel ou psychique», dans le contexte de la Loi sur l'aide et l'indemnisation des victimes d'actes criminels, ibid., art. $9\left(1^{\circ}\right)$.

49. Le législateur utilise désormais le mot «préjudice», de préférence à «dommage», pour désigner l'une des conditions du droit à réparation. Ce faisant, il évite que le mot «dommage» soit confondu avec l'expression «dommages-intérêts» (dont il est un diminutif dans le langage courant), lesquels constituent plutôt un effet de la responsabilité, c'est-à-dire un moyen de réparer le préjudice subi par la victime. Sur les différents sens possibles du mot «dommage(s)», voir Dictionnaire de droit privé, supra, note $2, v^{\circ}$ «dommage», sp. rem. $4^{\circ}$. 